\title{
eJRIEPS
}

Ejournal de la recherche sur l'intervention en éducation physique et sport

$17 \mid 2009$

Varia

\section{L'activité de l'élève en saut en hauteur : modélisation des connaissances-élèves en contexte scolaire de pratique}

\section{Claude Kermoal}

\section{(2) OpenEdition}

1 Journals

Édition électronique

URL : http://journals.openedition.org/ejrieps/5597

DOI : $10.4000 /$ ejrieps.5597

ISSN : 2105-0821

Éditeur

ELLIADD

Référence électronique

Claude Kermoal, «L'activité de l'élève en saut en hauteur : modélisation des connaissances-élèves en contexte scolaire de pratique », eJRIEPS [En ligne], 17| 2009, mis en ligne le 01 avril 2009, consulté le 18 mars 2021. URL : http://journals.openedition.org/ejrieps/5597 ; DOI : https://doi.org/10.4000/ ejrieps.5597

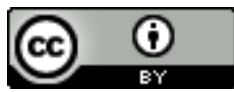

La revue eJRIEPS est mise à disposition selon les termes de la Creative Commons Attribution 4.0 International License. 
L'activité de l'élève en saut en hauteur : modélisation des connaissances-élèves en contexte scolaire de pratique.

Claude Kermoal, UMR-STEF, ENS Cachan et GRIAPS, UFRAPS-IUFM Besançon. Courriel : kermoalc@club-internet.fr

Résumé

L'objet de cette recherche de type exploratoire à orientation compréhensive est d'analyser l'activité produite par un élève lors d'une situation scolaire de saut en hauteur. L'orientation scolaire de la recherche organise le regard sur les pratiques familières des élèves comme objet en termes de rôles joués par les connaissances de l'élève et d'interrogation des conditions de leurs mobilisations au cours de l'action. La notion de Construction motrice personnelle englobe ces connaissances et les structures par des logiques d'actions liées à la position corporelle que l'élève pense pouvoir produire, caractéristique d'un type modélisable de franchissement de la barre.

La méthodologie s'appuie sur la mise en relation de deux modélisations de l'activité et articule action et espace subjectif d'action. Une première étude révèle la position de franchissement produite lors de 3 sauts réels par reconstruction $3 D$. Une deuxième analyse les discours sur l'action des élèves ayant sauté au cours d'entretiens de type auto-confrontation simple.

Les résultats obtenus montrent l'existence d'une catégorisation possible des sauts et de leurs caractères subjectifs en 3 modes d'action présents dans les trois classes de $5 e$ de collège ayant servi de population expérimentale (95 sauts analysés - 25 élèves).

Les politiques scolaires actuelles d'éducation sont organisées, dans leur grande majorité, autour de la construction et le développement des compétences de l'apprenant. Ce mouvement est marquant dans les dix dernières années aussi bien au niveau européen (Reiffers, 1997 ; Eurydice, 2002) que national, notamment dans les programmes scolaires d'éducation physique et sportive (EPS). La bascule qui s'est opérée vers 1990 passant de curricula centrés sur les savoirs à transmettre aux curricula centrés sur l'activité des apprenants marque un changement radical même si l'élève n'a jamais été véritablement absent des préoccupations institutionnelles et des recherches didactiques disciplinaires (Giordan \& Martinand, 1986). 
Alors que le cadre institutionnel incite à cette réorganisation, nous pouvons noter la faiblesse quantitative des publications portant sur l'activité de l'élève en situation de pratique scolaire. Les pratiques familières au sens donné par le laboratoire Sciences, Techniques, Enseignement, Formation (STEF) de I'ENS Cachan comme rapport particulier à l'enseignement que l'élève bâtit au cours de son expérience font encore l'objet de peu de publications. Peu de recherches se développent sur l'objet « activité de l'apprenant » en tant que construction d'actions motrices nouvelles et usage de connaissances par l'élève. Les travaux sur les conceptions et représentations de l'apprenant participent de cette orientation mais lorsque l'activité s'organise autour d'une forte composante motrice, ces concepts sont soumis à des nécessaires recontextualisations théoriques importantes dont le domaine de recherche des relations entre pensée et action tente la saisie (voir Bouthier, 1993, pour une synthèse). Dans les habiletés motrices exercées en milieu «stable » (Poulton, 1957), deux voies théoriques revendiquant un développement et une transformation de l'activité de l'apprenant coexistent dans les propositions didactiques scolaires en athlétisme; une liée à la construction de patrons gestuels adaptés aux contraintes de la situation (Schmidt, 1988) et l'autre s'appuyant sur la mise en situation pour générer une adaptation par développement de l'expérience (Durand, Saury \& Sève, 2006). La première voie s'appuie essentiellement sur les apprentissages de types procéduraux avec comme appui théorique une approche cognitiviste de la motricité envisageant l'apprentissage comme application de savoirs externes (Anderson, 1983) dans des situations de plus en plus contraintes. L'autre voie construit ses propositions sur l'authenticité des contextes situationnels comme conditions de mise en œuvre d'une motricité largement improvisée et réactive (Suchman, 1987), autorégulée (Temprado \& Montagne, 2001). Ces deux voies empruntent, pour chacune, l'intégration progressive de savoirs externes dans des situations aménagées et simplifiées, ou la mise en relation entre ressources disponibles du sujet et contraintes situationnelles authentiques et globales, mais en excluant par là même l'autre accès. Selon nous, elles se révèlent inadaptées pour appréhender «les itinéraires que doivent suivre les élèves pour pénétrer dans des territoires (...) qui n'ont encore pas été viabilisés » (Leclerc, 1994, cité par Lebeaume, 1999) en restreignant les acquisitions au temps scolaire et présent ou en masquant la question des conditions du développement des constituants de l'activité.

De récents travaux ont souligné l'importance d'une analyse en point de vue à la 1 ère personne (Vermersch, 2002) dans la production d'une activité athlétique en contexte (Gouju, 2001 ; Kermoal \& Gréhaigne, 2008). Cependant l'affirmation constituée autour d'un «primat accordé à l'intrinsèque » (Theureau, 2004, p51) privilégie un regard sur 
l'activité en termes d'interaction entre acteur-sujet et situation d'action (de type interactions en contexte, émergence, caractère situé de l'action ou encore couplage structurel). S'adressant à un acteur, principalement professionnel en situation de travail, cette approche construit l'objet « activité » dans une temporalité réduite à la situation et à ce qui y est « montrable, racontable et commentable par lui à tout instant » (Theureau, 2004). La dimension expérientielle y trouve une place dominante. Dans sa forme de présence dans les recherches sur l'intervention en STAPS, l'activité de l'acteur élève est analysée dans une dynamique intrinsèque à la situation. Elle occulte par là même le cadre ternaire de définition de la situation scolaire, constitué à minima par le savoir en jeu, l'enseignant et l'élève (Amade-Escot, 2007) et notamment sa constitution autour de contenus d'enseignement présents dans le curriculum de formation.

En dépassant la seule dimension expérientielle, la présente recherche prend comme objet les connaissances-élèves et les conditions de leurs mobilisations lors de la pratique effective dans une situation scolaire de saut en hauteur (dite «à barre montante »). Elle les appréhende en termes opératoires à partir des procédures réelles utilisées, et en termes subjectifs à partir des significations et des logiques présentes dans le discours sur l'action lors d'un revécu vidéo de ses propres sauts par l'élève. Bien que circonscrites par le curriculum de formation, ces connaissances appartiennent à l'interaction élève situation d'action. En s'installant par la pratique comme solution efficace et locale, elles caractérisent la nature de l'activité déployée par l'élève dans la situation. La question de la transformation de l'activité et des actions qui la constituent, renvoie directement à la confrontation entre les contenus d'enseignement, prescrits par l'apprentissage escompté et ces connaissances générant une efficacité locale et pragmatique. Lorsque le dispositif d'enseignement repose sur une simplification des contraintes portant sur l'action, ces connaissances peuvent être mises en sommeil temporairement mais elles réapparaissent lorsque les contraintes de la situation s'amplifient et peuvent se révéler ainsi comme des obstacles à l'apprentissage. Le concept de Construction motrice personnelle (CMP) agrège ces connaissances et leurs conditions de mobilisations dans une modélisation montrant le rôle central que joue la position de franchissement de la barre dans la construction du saut, la relative stabilité des constituants procéduraux et l'enracinement des opérations motrices dans une logique d'action. Elle aboutit à une catégorisation des sauts pour une tranche d'âge (classe de $5^{\text {ème }}$ de collège). 


\section{Cadre théorique}

Deux ensembles théoriques permettent d'appréhender les constituants des pratiques familières que les élèves développent pragmatiquement alors que leurs actions sont effectuées sous tutelle du prescrit curriculaire.

1. 1. La nature des savoirs curriculaires à construire par l'élève

Le premier élément du cadre théorique questionne la nature des savoirs définis comme visées curriculaires que la pratique athlétique scolaire, et plus particulièrement le saut en hauteur, installe comme constituants de la compétence et qu'il convient de mettre à jour pour appréhender la spécificité de notre objet de recherche. Cette voie ne saurait cependant épuiser les contenus dont la discipline d'enseignement, considérée dans son support athlétique saut en hauteur, est porteuse (Martinand, 2001). La pratique sociale du saut en hauteur fournit des constructions techniques et des modes d'actions privilégiés, générant un ensemble de pratiques et de savoirs objectivés, dans lequel la discipline EPS puise ses références.

La complémentarité de ces deux premiers éléments théoriques permet d'objectiver, ce que nous emprunterons à Pastré (2005) en l'adaptant aux caractéristiques scolaires, les façons de faire sollicitées par la situation sur un plan épistémique, en dehors de ses composantes singulières et individuelles. La modélisation cognitive qui en ressort, met à jour les organisateurs de l'activité scolaire de saut en hauteur autour de trois pôles de détermination : la performance individuelle et/ou collective, la maîtrise des procédures en fonction des ressources individuelles et le projet d'action installant une réflexion sur sa propre action.

L'activité de saut en hauteur peut être quant à elle décrite dans la coordination de deux secteurs d'action (Kermoal, 2008) :

- l'élévation du corps par-dessus la barre qui envisage la trajectoire aérienne du corps, la position du point-mort haut, ... Liée aux qualités physiques et à la taille du sauteur, l'élévation absolue peut être relativisée par la différence entre hauteur maximale du bassin lors de la phase aérienne et sa hauteur initiale ou encore référée à la hauteur de la barre à franchir.

- la position du corps et ses variations, pour qu'aucune partie du corps ne touche lors du franchissement de la barre. Cette position peut être objectivée dans sa dynamique de production lorsque les rotations du corps amenant à cette position sont prises en compte, le terme de posture sera utilisé. Selon Dapena (1980), les rotations dans le saut en Fosbury sont de deux types: une rotation selon le grand axe du corps qui amène le sauteur dos à la barre (twisting rotation) et un ensemble de rotations qui amène le sauteur 
à plat par-dessus la barre (somersaulting rotation). La figure 1 présente ces éléments de modélisation épistémique de l'acte de saut.

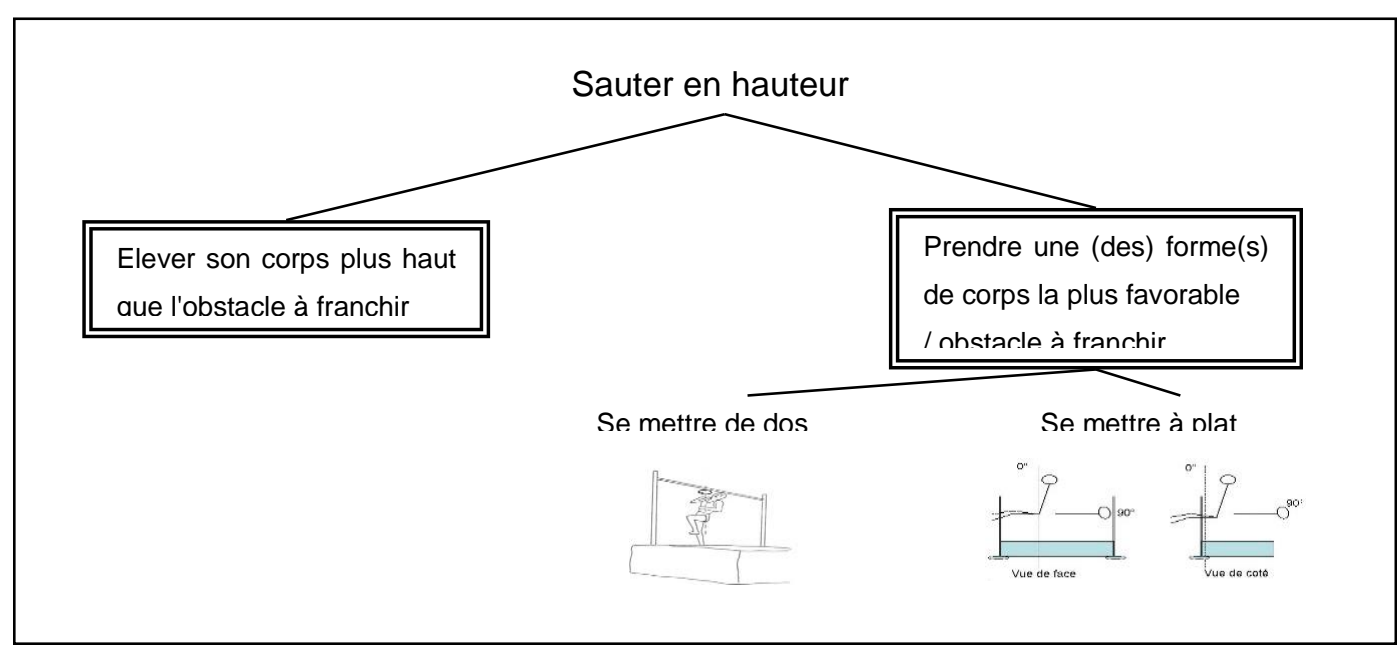

Figure 1. Modélisation épistémique de l'acte de saut

1. 2. Les connaissances élèves et les conditions de leurs mobilisations

En soulignant la nature diverse des connaissances que l'élève investit dans son acte (Gréhaigne, Wallian \& Billard, 2005), un second ensemble théorique s'appuie sur les concepts de représentation pour l'action (Weill-Fassina \& al., 1993) et de conception des apprenants (Giordan \& al., 1994) pour interroger les relations entre la situation et les connaissances construites et stabilisées par l'élève. Alors que la conception fonctionne sur un registre explicatif extérieur à l'action motrice, elle lui fournit une organisation mentale permettant le décodage préalable de la situation et l'élaboration de liens entre les faits produits et les évolutions envisageables de l'action. La représentation pour l'action, quant à elle, est directement opérative mais son caractère indépendant à l'égard de la situation tel que le paradigme du Traitement de l'Information l'envisage est d'ailleurs remis en cause par les travaux produits par la didactique professionnelle (Rogalski, 2004). Ces derniers appuient sur le rôle du contexte dans la mobilisation des connaissances, notamment au travers des propositions de Borghi (2004). L'existence d'une temporalité interne, reconstruite par le sujet pour pouvoir agir a été avancée par Gouju (2002). Ces données constituent un point d'ancrage pour une prise en compte des représentations pour l'action et des conceptions comme facteurs constituants de l'activité athlétique de l'élève.

Le dernier point du cadre théorique envisage le schème d'action comme «forme d'organisation invariante de l'activité »(Vergnaud, 1998). Les quatre composantes du 
schème d'action proposées par Vergnaud permettent de définir le contenu de la forme opératoire utilisée par l'élève pour sauter en hauteur :

- le but, les sous-buts, et les anticipations fixent les motivations à agir de l'action, - les règles d'action, de prises d'informations et de contrôle sont les éléments de structuration de la logique opératoire présidant à l'engagement du sujet dans la situation, - les invariants opératoires ou concepts-en-acte jouent à la fois le rôle d'opérateur dans la situation mais également de spécification de l'environnement dans la mesure où ils traduisent la situation présente en fonction des modes opératoires disponibles chez le sujet,

- les inférences installent l'action dans une organisation la dépassant en fixant des voies d'évolution et des paramètres d'action.

Cette proposition a été utilisée par Pastré (2004) pour mettre à jour le rôle du schème d'action dans des activités à visée pragmatique et par Recopé (1996) pour montrer son organisation fonctionnelle et signifiante chez le volleyeur sportif.

Ces éléments théoriques nous permettent d'avancer l'idée de la mise en œuvre d'une activité signifiante organisant l'acte de saut autour d'une construction du saut en appui avec ce qui semble à l'élève devoir faire pour franchir la barre. Lorsque la hauteur de la barre pose un problème de franchissement au sauteur, les savoirs curriculaires peuvent alors entrer en interférence avec l'efficacité locale portée par ce schème d'action.

\section{Méthodologie}

La méthodologie employée emprunte à Pastré (2005) son versant opératoire qui s'intéresse aux façons de faire effectivement utilisées par les élèves dans la situation de saut. Cette partie de modélisation opérative articule deux regards sur l'activité du sujet : d'une part, sur les opérations constituant l'acte de saut dans son versant opératif et, d'autre part, sur les connaissances mobilisées par les élèves pour commenter leurs sauts constituant le versant subjectif.

2. 1. Les données de recherche de type opératoire : la position du corps lors du franchissement

Une méthodologie de reconstruction du saut en 3D est utilisée pour rendre permanentes les données de recherche de type déplacement du corps, positions et déplacements des différentes parties en jeu dans l'activité scolaire de saut. Le logiciel 3D vision (Dietrich, $1995)$ est employé pour traduire en coordonnées dans un espace ( $X Y Z$ ) les données cinématiques du mouvement issues de trois caméras synchronisées manuellement filmant le même saut (voir Figure 2). 9 points-corps sont saisis pour chacune des trois caméras 
$(25 \mathrm{mhz})$ lors des trois dernières foulées et sur la trajectoire aérienne. Un référentiel fixe par rapport au sautoir $(X, Y, Z)$ est utilisé. Les coordonnées des éléments matériels du sautoir sont connues dans ce référentiel $X Y Z$. 3 sauts par élève sont été saisis et reconstruits par 3D vision - sauf exceptions liées notamment à des masquages importants de point-corps - et 28 élèves répartis sur deux sites de passation. L'enregistrement a eu lieu lors de la 2 e ou 3 e séance selon les sites. A partir de la hauteur maximale franchie précédemment (hauteur maximale «R»), les sauts $S 1$, $S 2$ et $S 3$ ont été réalisés à des hauteurs définies par $\mathrm{S} 1: \mathrm{R}-15 \mathrm{~cm}, \mathrm{~S} 2: \mathrm{R}-5 \mathrm{~cm}$, et $\mathrm{S} 3: \mathrm{R}$.

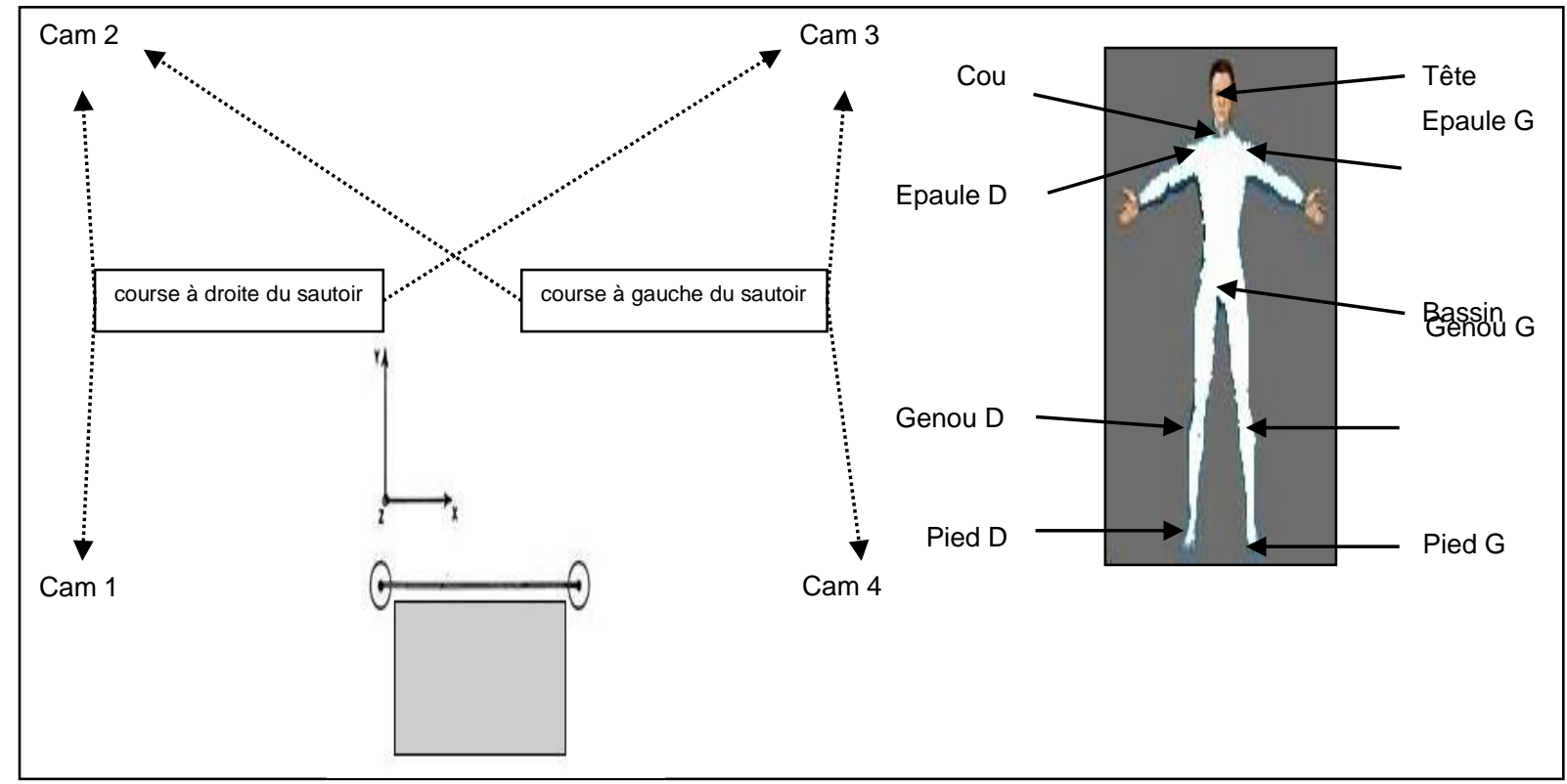

Figure 2. Dispositif de recueil des données opératoires

La position de franchissement sera décrite à partir de deux marqueurs : la position de l'axe des épaules lorsque le bassin franchit la barre (Marqueur [A1éptBAR]) sur une vue aérienne, la position du tronc par rapport à la verticale à ce même moment (Marqueur [A2cbtBAR]). Ces deux données de recherche sont exprimées en degré.

Le marqueur [A1éptBAR] décrivant la position de la ligne d'épaules est une mesure indirecte de la position du tronc lors du franchissement dans un plan horizontal (vue aérienne). Le choix de cette variable est effectué pour éviter les erreurs de mesure de la position du tronc lorsque celui-ci est vertical. Dans ce plan, les points-corps cou et bassin sont en effet quasiment alignés attachant la mesure d'erreur importante. Les valeurs de référence pour [A1éptBAR] sont la perpendiculaire au grand axe de la barre - fixée à $270^{\circ}$ - le tronc est à cette valeur parallèle à la barre, si on considère que la ligne d'épaules est perpendiculaire au grand axe du tronc et la valeur $360^{\circ}$ indiquant une ligne d'épaules 
parallèle à la barre et donc un tronc perpendiculaire à celle-ci. Une vérification visuelle sur la bande vidéo est effectuée pour extraire de la population expérimentale les sauts dans lesquels la situation de la ligne d'épaules se différencie fortement de la perpendiculaire du tronc.

Le marqueur [A2cbtBAR] quantifie l'inclinaison du tronc par rapport à la verticale. Comptetenu de l'existence d'une modification de l'orientation globale du corps - quantifiée par [A1éptBAR] - pour certains types de franchissement en saut en hauteur, l'inclinaison du tronc par rapport à la verticale pose également un problème de mesure. En effet, elle peut apparaître dans un certain plan de représentation - par exemple plan YoZ - et pas dans le plan qui lui est perpendiculaire - plan XoZ, dans cet exemple. Pour palier cette difficulté de calcul, le choix est effectué de passer dans un système de coordonnées sphériques permettant l'expression de l'inclinaison quelle que soit l'orientation du tronc dans l'espace.

2. 2. Les données de recherche de type subjectif : les discours sur l'action

II s'agit d'accéder à ce niveau à la partie orientatrice de l'action (Léontiev, 1972), celle qui fait que, selon notre cadre théorique, le mouvement humain s'organise comme une mise en œuvre de moyens en fonction d'un but. L'hypothèse du présent travail envisage la position corporelle de franchissement et les déplacements aériens du corps pour la produire comme finalités des connaissances mobilisées par l'élève autour de son saut. L'analyse des significations et des logiques mises en œuvre dans l'action se fait par une adaptation de la méthode par entretien d'auto-confrontation (Theureau, 2004) à partir d'une trace vidéo des sauts, caméra « à l'épaule ». Cette caméra est ajoutée au dispositif décrit précédemment. Treize élèves du deuxième site ont participé à cette phase en fonction de leurs positions de franchissement observées. Les entretiens ont eu lieu durant le cours d'EPS de la semaine qui suit l'enregistrement vidéo. Ils demandent à l'élève regardant ses sauts à vitesse réelle ou ralentie, de situer en revécu subjectif les moments qui lui semblent importants pour la réussite ou l'échec de son saut. Six moments sont définis à partir des entretiens : la préparation du saut, la course d'élan, l'approche de la barre, le décollage, le franchissement et la réception du saut. La possibilité de revenir en arrière ou d'arrêt sur image est offerte. Des relances d'entretien ont systématiquement lieu lorsqu'un moment est identifié pour accéder à la nature des actions motrices subjectivement présentes à ce moment et $\mathrm{au}(\mathrm{x})$ partie(s) du corps, siège(s) de l'action. L'analyse des données de verbalisation se fait par méthode qualitative et vise à objectiver les connaissances que l'élève mobilise pour expliquer et commenter sa prestation.

La recherche teste la stabilité des CMP mises en pratique par les élèves dans les conditions de confrontation à une situation authentique de franchissement de barre en 
saut en hauteur puis met en parallèle deux types de données de recherche, les modes opératoires et les constituants de discours sur l'action, pour définir les ressorts de l'activité produite par l'élève en situation de saut en hauteur à hauteur de barre proche de son record.

\section{Résultats}

95 sauts ont été analysés pour une population de 25 élèves - 3 sauts réels, pour la majorité des élèves, et un 4ème reconstruit par les moyennes des valeurs obtenues pour les marqueurs [A1éptBAR] et [A2cbtBAR]. 3 élèves ont été exclus de la population initiale $(n=28) d u$ fait de leurs sauts atypiques rendant impossible l'application des variables d'analyse définies. Après validation de la stabilité des positions produites, le 4ème saut servira de lissage et d'artifice de figuration des données de recherche. Les sauts ont été analysés quel que soit la réussite ou l'échec du franchissement à partir du moment où l'échec n'est pas consécutif à une dégradation signifiante du comportement. Ainsi, certains sauts ont été exclus du plan expérimental et remplacés par d'autres lorsque cela était possible.

Il convient de noter que le mode de constitution de la population expérimentale interdit l'étude d'une répartition quantitative ; l'ensemble des élèves du 1er site (10 élèves - 39 sauts) a fait l'objet d'une saisie donnant un profil de répartition pour les marqueurs [A1éptBAR] et [A2cbtBAR]. Le 2e site a permis d'alimenter en effectif les groupes observés par la sélection d'individus représentatifs (15 élèves - 56 sauts). Afin de ne pas limiter les observations à celles effectuées à partir du 1er site, les élèves du 2e site ayant des valeurs divergentes ou extrêmes par rapport aux premières observations ont été systématiquement retenus dans la population expérimentale.

\section{1. La catégorisation des positions de franchissement observées}

La figure 3 représente les valeurs observées pour les deux marqueurs objectivant la position au moment du franchissement de la barre (Marqueurs [A1éptBAR] et [A2cbtBAR]). Avec une représentation graphique de type $X Y$, un relatif étalement des sauts peut être constaté sur l'axe des abscisses caractérisant la position des épaules. Les valeurs varient de $232^{\circ}$ (BR135) - par convention, [BR135] indique le saut de l'élève identifié (BR) et la hauteur de barre de ce saut (135) - à $372^{\circ}(\mathrm{SX120})$ ce qui correspond à une position corps $3 / 4$ face à la barre (BR135) et perpendiculaire à celle-ci (SX120). Les valeurs d'inclinaison du tronc par rapport à la verticale quelle que soit son orientation dans 
le référentiel $(X Y Z)$ varient entre $8^{\circ}$ (LG110) et $74^{\circ}$ (GA125) ce qui correspond à un tronc quasi vertical (LG110) et presqu'horizontal (GA125).

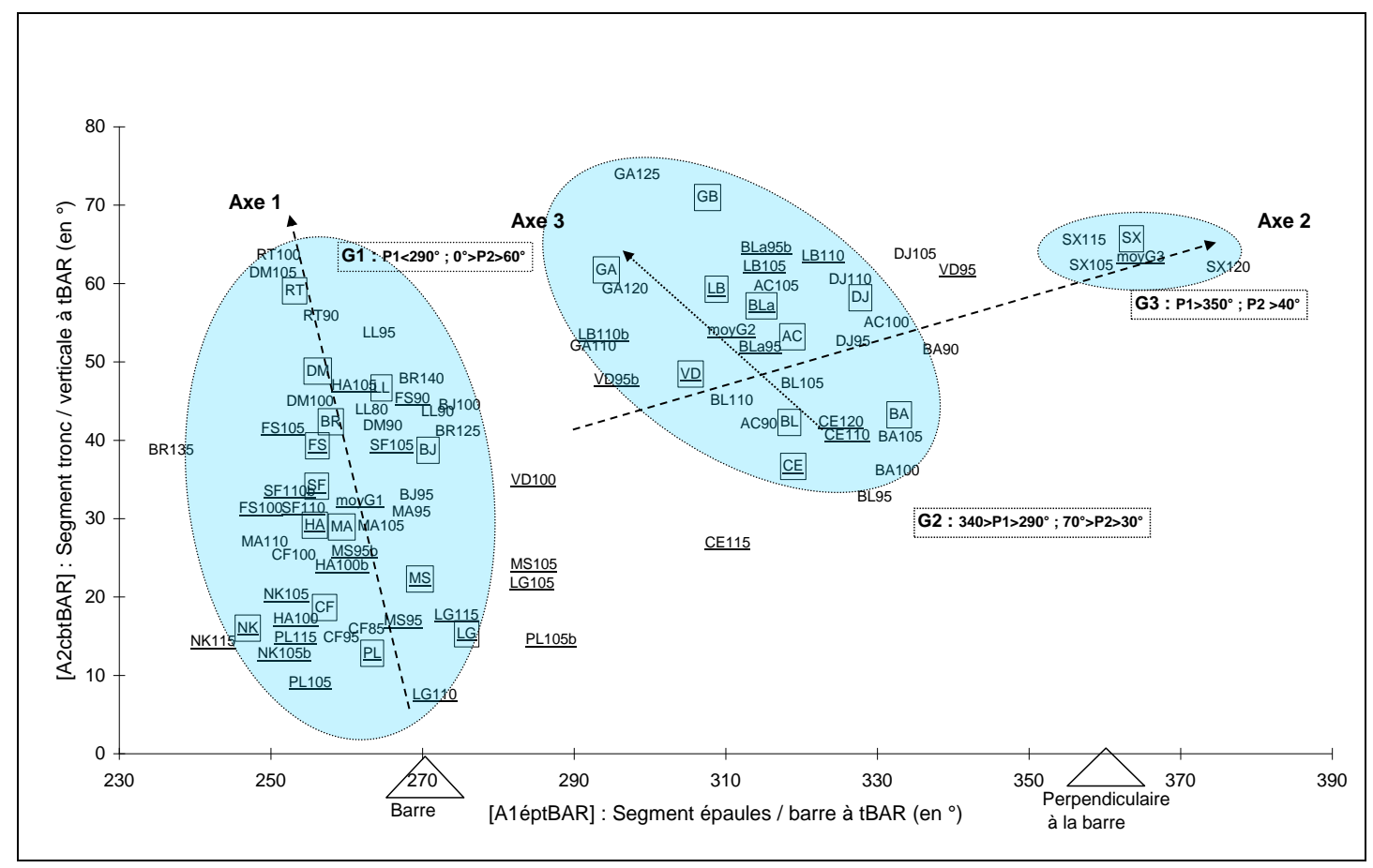

Figure 3. Positions de franchissement observées et catégorisation en 3 groupes.

Les sauts encadrés figurent le $4 \mathrm{e}$ saut dit «moyen ». Par convention, MA95 identifie l'individu MA et la hauteur de barre tentée .Les individus appartenant au 1er site sont soulignés.

Deux axes principaux organisent le graphique :

un axe vertical reliant les valeurs les plus faibles de [A1éptBAR] entre 250 et $270^{\circ}$ avec une augmentation progressive de [A2cbtBAR] jusqu'à 60 , noté Axe1

un axe incliné reliant [A1éptBAR] avec une croissance progressive de [A2cbtBAR], noté Axe2.

Trois regroupements peuvent être observés sur la Figure 3 :

Groupe 1 (G1) regroupant 11 élèves BJ, BR, CF, DM, FS, HA, LL, MA, MS, PL et RT et est caractérisable par une évolution de [A2cbtBAR] alors que [A1éptBAR] reste stable. L'observation de [A1éptBAR] indique un tronc parallèle à la barre, et [A2cbtBAR] montre une inclinaison progressive par rapport à la verticale.

Groupe 2 (G2) pour les 10 individus AC, BL, Bla, CE, DJ, GA, GB, LB, LG et VD. [A1 ép tBAR] est supérieur à $290^{\circ}$ indiquant un tronc orienté entre 30 et $50^{\circ}$ par rapport à la barre. L'inclinaison du tronc par rapport à la verticale évolue en sens opposé, - axe 3 de Figure 3. Deux sous-groupes extrêmes (BA, BL, CE) et (GA, GB) apparaissent alors.

Groupe 3 (G3) regroupant un seul individu (SX) et se situant dans la continuité des valeurs constatées pour G2. Par rapport à ce dernier groupe, seule une valeur de [A1éptBAR] plus 
élevée pour ce groupe G3 est observable. L'existence d'un « trou » entre les valeurs pour les groupes G2 et G3 peut faire l'objet d'une interrogation, l'hypothèse d'un artefact expérimental lié à la population observée ne pouvant être exclue.

3. 2. Les constituants des discours sur l'action - schèmes d'action

L'analyse des discours sur l'action vise à mettre au jour les constituants subjectifs engagés par l'élève dans son activité. Dans le cadre théorique, nous avons avancé l'idée d'une construction du saut dans un espace singulier reposant sur les propriétés que le sauteur prête à la situation et sur les actions et ressources qu'il lui semble pouvoir mobiliser. La grille d'analyse mobilisée par l'élève pour commenter sa propre prestation permet, selon notre hypothèse, l'accès aux connaissances individuelles définissant cet espace singulier. Cette construction s'inscrit dans une temporalité élargie aux différents moments du saut et autour de celui-ci. Elle s'exprime dans les buts, sous-buts et inférences que le sujet exprime pour analyser son action dans les conditions d'entretien d'auto-confrontation adapté au cadre didactique de la recherche.

Les discours sur l'action sollicités dans cette recherche ont fait l'objet d'une définition en termes d'Espace Subjectif d'Action -noté ESA- (Kermoal \& Gréhaigne, 2008). II s'agira lors de cet article de mettre en relation ces ESA avec les caractéristiques motrices du franchissement opéré par le sauteur pour appréhender les constituants de schèmes d'action spécifiques isolables dans les pratiques familières aux élèves à des moments particuliers de l'apprentissage. Trois schèmes d'action peuvent ainsi être décrits à partir des moments et de types d'actions envisagés comme licites et des caractéristiques de la position de franchissement observables en situation.

3. 2. 1. ESAa - le lancement de parties du corps au dessus de la barre Les unités caractéristiques de ce schème d'action sont essentiellement situées dans les moments « décollage » et «élan ». Les actions utilisées décrivent le décollage comme un lancer ou une levée de la jambe libre - par convention, la jambe « libre » est celle qui n'est pas en appui au sol lorsque le sauteur décolle. La jambe en contact avec le sol à ce moment sera nommée jambe «d'appel». Aucun ancrage d'action au sol de la jambe d'appel, liée par exemple à une poussée, ne figure dans les entretiens. Par la suite du saut, la jambe d'appel sera mobilisée dans une action assurant son retour lors du franchissement de la barre. Le franchissement et le décollage se trouvent ainsi confondus dans une même série d'actions mobilisant principalement les membres inférieurs. Cette centration alternative sur chaque pied, évoque sans que cela apparaisse nommément, un franchissement de type saut en ciseau et donne priorité au pointage locomoteur d'un point du corps, le pied ou le genou libre par-dessus la barre au moment du décollage : « je lève 
jusqu'à ce qu'elle dépasse la barre » [LL044] - ([LL044] indique le numéro de réplique dans l'entretien réalisé avec l'élève LL).

Secondairement, l'action décrite dans le terme «viser» provoque une organisation particulière de la course d'élan qui présente une volonté de retenue liée soit à une peur, ne pas retomber sur le tapis (CF), soit aux contraintes liées au pointage de la jambe libre (LL).

Trois individus présentent les caractéristiques de cette forme de discours : BJ, CF et LL auxquels il conviendrait sans doute d'ajouter MA, qui présente une forme mixte entre ESAa et ESAb.

3. 2. 2. ESAb - la prise d'une position favorable sur une trajectoire

La projection du corps par-dessus la barre est le moment dominant dans le discours caractéristique de cet ESA. II correspond à une recherche d'une position favorable de franchissement dans laquelle tout ce qui dépasse du corps pourra être rentré (« mettre les 2 jambes ensemble » [DM018]). Cette position fait apparaître le moment du franchissement de la barre comme succédant temporellement au moment de décollage qui est organisé par un champ lexical spécifique (« s'étirer » [RT040], « me jeter en haut » [FS012], ...). L'autonomie relative de ces deux moments permet la construction d'un décollage alors libéré des contraintes de précision du pointage locomoteur, qui peut s'inscrire dans une projection véritable ("j'essaie de pousser mon corps vers le haut" [FS083]) dans laquelle la vitesse d'élan joue un rôle essentiel ("là je mets la puissance" [FS054]) comme règle d'action et comme inférence sur la situation ("plus la barre monte, plus je cours vite" [DM012].

Les discours sur l'action tenus par trois individus correspondent aux caractéristiques décrites : DM, FS, RT. MA, cité précédemment pourrait également figurer dans ESAb.

3. 2. 3. ESAC - l'engagement sur le dos - épine dorsale du saut

Six individus peuvent être considérés dans cette forme identifiée ESAc : BA, BL, DJ, GA, GB, SX. Cette troisième forme de discours sur l'action se caractérise par la présence d'un nouveau mode d'action licite dans le discours. Son contenu est retranscrit par des verbes indiquant la mise en rotation du corps («pivoter» [GB006], « se retourner» [BL048], « tourner ses épaules» [DJ016]). Le corps subit par ce mode d'action un changement de face (« se mettre de dos » [SX026]) qui apparaît soit au décollage, lors du franchissement de la barre ou à la réception sur le tapis. Le décollage se trouve ainsi « envahi » par une double intention, monter et tourner qui peut s'envisager comme un problème. Le contenu opératoire d'un des deux thèmes peut disparaître alors des actions décrites lors du décollage, par exemple GA. 
Les parties du corps évoquées lors des entretiens se situent essentiellement au niveau du haut du corps (épaules, dos) avec parfois des mentions à l'action de poussée de la jambe ou du pied d'appel (SX).

3. 3. La stabilité des positions de franchissement produites

Alors que l'activité non-experte est classiquement décrite comme erratique et inconstante, l'analyse des données montre une stabilité des positions de franchissement observées pour les 25 élèves de la population analysée. L'hétérogénéité de constitution des données de recherche rend délicate l'utilisation d'un test statistique standardisé étudiant la similarité des valeurs pour les marqueurs [A1éptBAR] et [A2cbtBAR]. Ces valeurs proviennent, en effet de données appariées si on considère les valeurs obtenues par un même élève durant ses quatre sauts et de données indépendantes si on considère chaque élève par rapport à l'ensemble de la population expérimentale. Compte tenu de ces difficultés méthodologiques, la stabilité de la position de franchissement est étudiée au moyen du calcul des écarts au saut moyen (4e saut reconstruit) des valeurs produites pour chaque marqueur dans chacun des sauts (sauts réels). La moyenne obtenue des écarts au saut moyen est pour [A1éptBAR], 6,8 (écart-type 6,125) et pour [A2cbtBAR], 5,0 (Ec-T $3,792)$. Ces données sont représentées en Figure 4 qui reprend le mode de constitution de la Figure 3. L'ensemble des sauts effectués par les 2 élèves présentant les écarts au saut moyen les plus élevés pour chacun des 2 marqueurs y est représenté (soit 4 élèves au total). Les positions graphiques observées, bien que présentant des différences, ne remettent pas en cause la catégorisation que nous avons produite lors de l'analyse des positions de franchissement. Elles permettent d'attester la présence d'invariances dans les sauts produits par un même élève, observés par leurs effets, tout en laissant la place à une marge de variabilité permettant sans doute à l'action d'évoluer dans des circonstances particulières, produites en situation de saut par l'élève ou fortuites. Ce résultat montre que les caractéristiques d'invariance du schème d'action, décrites par Vergnaud (1998) dans des activités mathématiques, se retrouvent dans les actions motrices produites en cours d'apprentissage du saut en hauteur. II organise le rôle central que joue la position de franchissement dans une modélisation des actions et des connaissances du sauteur. 


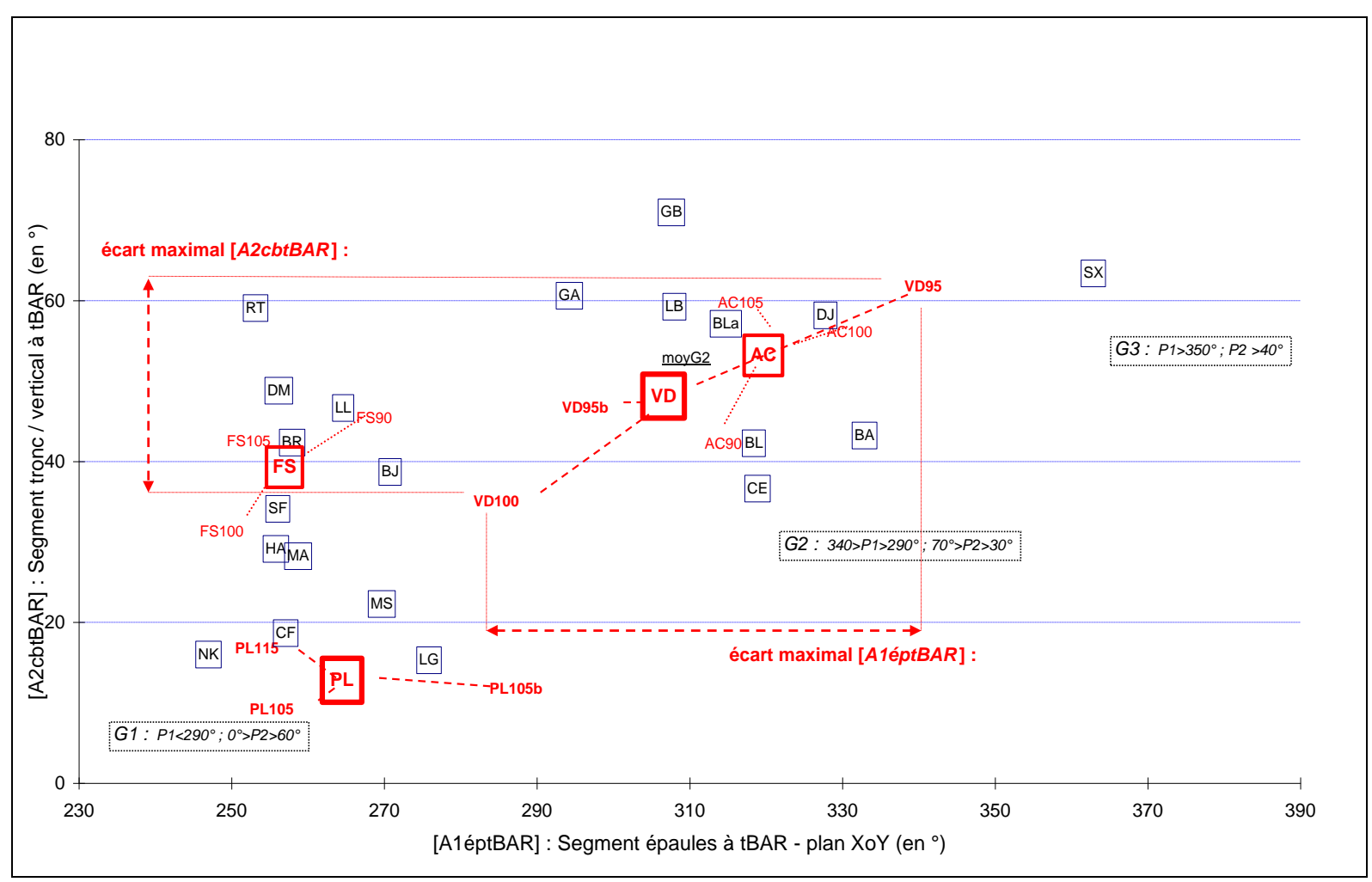

Figure 4. Positions extrêmes des marqueurs pour les 4 individus (pour chaque marqueur les deux sauteurs présentant la dispersion la plus forte).

3. 4. La correspondance entre position de franchissement et discours sur l'action La figure 5 met en relation la position observée de franchissement, suivant les caractéristiques décrites dans la figure 3 et la catégorisation effectuée du discours sur l'action en trois ESA spécifiques. Cette représentation vise à mettre en évidence la correspondance entre les caractéristiques de la position de franchissement, finalisant l'activité motrice développée, et les éléments dominants du discours sur l'action, permettant l'accès aux modes d'action reconnus comme licites par l'élève. Ce lien établi entre ces 2 niveaux de lecture de l'activité humaine permet d'appréhender le schème d'action comme outil théorique pour expliquer la production motrice en situation d'apprentissage.

Selon la figure 5, une relation de proximité peut être établie entre des positions corporelles produites et les éléments présents dans le discours sur l'action. Les regroupements effectués pour caractériser les positions de franchissement coïncident en partie avec les formes identifiées de discours sur l'action. 


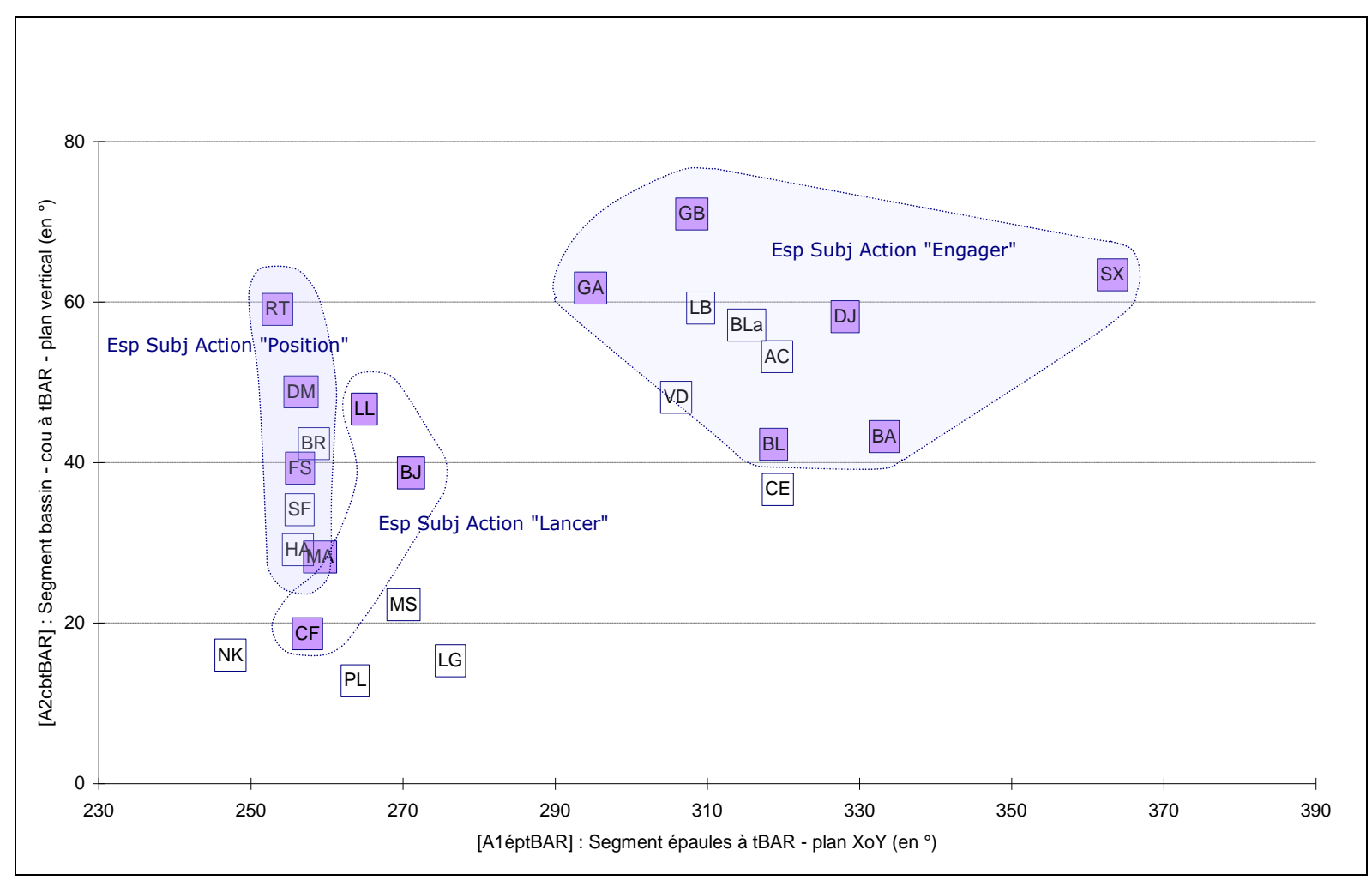

Figure 5. Représentation des espaces subjectifs d'action vs. positions de franchissement

- Les groupes de position de franchissement G2 et G3, dont nous nous sommes demandé si la partition n'était pas un artéfact expérimental, développent principalement dans le discours des modes d'action licites sur la thématique de l'engagement du corps dos à la barre ou du retournement-rotation lors du décollage. Observable chez les différents élèves constituant ces 2 groupes et ayant passé les entretiens, cette thématique de l'engagement corporel coïncide avec une variation observée des valeurs de [A1éptBAR]. Ceci montre un lien effectif entre les effets produits par les actions motrices réelles et les buts poursuivis dans la construction subjective du saut. Ce résultat est renforcé par le fait que l'absence d'un engagement de cette nature dans le discours provoque l'absence de modification des valeurs observées de [A1éptBAR].

- L'effectif du groupe G1 se répartit entre deux ESA qui occupent alors des positions proches sur la répartition graphique. Suivant le marqueur [A1éptBAR], la position de franchissement du groupe G1 est caractérisée par une absence de modification de l'orientation du corps entre la position au décollage et la position de franchissement moyenne des rotations observées, par ailleurs, est 5,8 (Ec-T 14,37) -. Nous pouvons mettre en relation cette organisation du décollage par la verticalité du tronc avec la prédominance dans le discours d'action de thématiques de type "lancer-viser une partie du corps par-dessus la barre » ou de type "projection + prise de position favorable ", 
telles qu'elles sont présentes dans ESAa et ESAb. En effet, la fixation du tronc sur l'axe vertical lors du décollage peut remplir deux fonctions dans l'organisation du saut. Soit elle permet d'assurer une précision au lancer-viser en limitant l'évolution dynamique du corps par rapport à la barre lors du décollage, suivant le principe de fixation de degrés de liberté (Davids, Handford \& Williams, 1994 ; Temprado \& Montagne, 2001). Soit elle produit les conditions mécaniques d'une projection du corps efficace, en organisant un axe de force lors du décollage (Piron, 2008).

- La mise à plat du corps lors de la phase aérienne, observable par le marqueur [A2cbtBAR], se répartit entre les différents groupes G1, G2 et G3. Elle ne peut pas être caractérisée de manière exclusive par un constituant particulier de discours sur l'action mais entretient sans doute des relations complémentaires avec les différentes thématiques extraites. Pour G1, le lancer-viser de la jambe ou du pied libre a des conséquences plus ou moins importantes sur la position verticale aérienne du tronc en fonction de la hauteur relative de la barre par rapport au corps du sauteur, et notamment par rapport à sa taille. Plus la barre est haute par rapport au corps, plus la visée va nécessiter une bascule du tronc vers l'arrière, qui apparaîtra lors du décollage ou postérieurement à celui-ci. Cette observation permet de comprendre les caractéristiques spécifiques de sauts effectués à des hauteurs identiques, tels que ceux de [CF] et de [LL], qui, de taille plus petite, doit incliner plus son tronc vers l'arrière pour assurer le pointage du pied libre revendiqué dans le discours d'ESAa. Pour un second groupe appartenant à G1, l'évolution observée des valeurs d'inclinaison du tronc traduisent également une bascule de celui-ci vers l'arrière. Cette bascule organise, selon les modalités d'ESAb, une prise de position dans laquelle la centration subjective plus ou moins prégnante sur le franchissement des membres inférieurs et l'apparition des actions de franchissement durant le déroulement du décollage, vont intervenir. Pour G2 et G3, le différentiel de valeur observé pour [A2cbtBAR] se réduit par la disparition des valeurs d'inclinaison les plus basses. Liée à la thématique d'engagement du corps par-dessus la barre, la bascule observée du tronc par rapport à la verticale pour ce groupe, est provoquée par une recherche volontaire d'une position allongée lors du franchissement de la barre. Elle est reliée soit à une organisation verticale lors du décollage, ou de manière fréquente à une inclinaison du tronc chez le sauteur non-expert dès le décollage (Pauly, 2002). Cependant elle permet de relier dans la construction du saut, retournement et mise à plat du corps lors du franchissement.

Ainsi les trois ESA mis en évidence lors de l'analyse des discours sur l'action renvoient à des structures motrices particulières observables lors du franchissement. L'articulation 
entre les effets de l'action et les structures mentales mobilisées par le sauteur pour commenter sa propre action permet d'affirmer l'existence d'une cohérence entre les constituants mentaux d'un acte-en-projet et leurs mises en œuvre effective en situation, ce qui renvoie directement aux caractéristiques du schème d'action tel qu'il est développé par Vergnaud (1998). De telles conclusions ont été aussi mises en évidence par Récopé (1996) dans l'analyse des comportements de joueurs non-experts en Volley-ball.

\section{Discussion et conclusion}

Les résultats produits par cette recherche permettent de pointer un certain nombre de caractéristiques spécifiques de l'activité athlétique déployée par le pratiquant scolaire. En prenant comme support une situation scolaire typique de pratique, largement utilisée lors des évaluations, ils montrent l'existence d'une voie pragmatique de développement de connaissances ayant une efficacité locale, liée à la réussite de sauts à des zones de hauteurs de barre individuellement déterminées. Ils montrent également l'intérêt d'appréhender, à l'aide du concept de schème d'action, l'activité athlétique de l'élève comme une organisation globale non réductible à une suite d'opérations motrices mobilisables indépendamment d'un contexte authentique de pratique.

4. 1. Les caractéristiques de la confrontation élève situation de performance données de l'implémentation curriculaire

Alors que la perception classique du curriculum en EPS s'appuie sur une construction par implémentation de savoirs à partir d'allers-retours entre situations réduites et situations « réelles » de pratique, les résultats de cette recherche tendent à montrer que la confrontation de l'élève à cette situation « réelle » est elle-même pourvoyeuse de connaissances, qui ne peuvent être seulement considérées comme des erreurs par la surface d'efficacité locale qu'elles autorisent, notamment aux hauteurs de barre franchies en utilisant la structure d'action alors présente chez l'élève. Nous avons nommé ces structures, Constructions motrices personnelles, pour en affirmer l'orientation opératoire (Kermoal, 2008). Elles ont un véritable fondement opératoire dans la mesure où elles entretiennent à la pratique une relation d'efficacité et articulent aux configurations perçues de la situation - le franchissement de la barre notamment pour le saut en hauteur - l'usage de moyens personnels mobilisables pour produire le but escompté au sein d'un acte-enprojet. Cette construction apparaît dans la situation lorsque la motricité est sollicitée dans de fortes contraintes situationnelles, où l'investissement énergétique et la performance doivent être maximaux. Dans ces caractéristiques, la situation fournit à l'action un contexte «authentique » au sens où elle sollicite pour le sauteur, une coordination spécifique de 
plusieurs secteurs d'action, élévation et positionnement aérien du corps notamment selon les termes de la modélisation cognitive que nous avons fait figurer en 2.1, qui génère l'appel à une construction mentale préalable de l'action.

La CMP tente de saisir ces constituants de l'activité de l'élève en montrant sa structure logique et les liaisons qui s'établissent à la pratique. Le rôle de la cognition individuelle s'y trouve mis en jeu et la question des relations entre cognition et action ne peut manquer d'être posée. Lors d'une recherche précédente, Barrué (1994) a montré le décalage pouvant exister entre le projet d'action déclaré par l'élève et la réalisation motrice. Avec la $\mathrm{CMP}$, le présent travail envisage ces relations entre cognition et action non comme un guidage cognitif des procédures mises en jeu dans l'action mais comme un arrière-plan construit à partir de connaissances existantes, extraites d'autres activités motrices ou mentales, d'une histoire personnelle ou partagée, qui vont fournir un contexte singulier à l'action de l'élève. Selon nous, cet arrière-plan par lequel l'acte de saut s'installe, peut entretenir des conflits d'apprentissage lorsque les visées curriculaires chargent la situation d'un contenu éloigné de la structure d'action actuellement mise en jeu par l'élève. La nature de ces conflits permet alors la caractérisation de la situation d'enseignement comme un champ d'études dans lequel le savoir curriculaire devrait prendre place en obtenant un statut opérant (Amade-Escot, 2007), mais dont la licité peut très bien être remise en cause lorsque son acquisition nécessite de rompre avec les logiques opératoires mises en jeu dans l'acte efficace.

Ces caractéristiques contextuelles sont sans doute particulièrement présentes dans les activités athlétiques par la dimension performance maximale requise, mais elles questionnent plus largement l'ensemble des activités support de l'EPS.

4. 2. L'identité individuelle et le développement de compétence scolaire La CMP avance l'idée d'une activité singulière naissant d'un arrière-plan que la situation par la hauteur de barre notamment - appelle en fonction de ses caractéristiques locales ou générales. La démonstration de relations entre les éléments représentationnels, les règles individuelles que l'action installe et la mobilisation d'actions motrices spécifiques pour passer la barre, renvoie à l'idée déjà développée par Gouju (2001) d'une identité du sujet. Elle place également l'acte de saut dans une dimension singulière et située, en contexte tel qu'il existe pour le sauteur au moment de son action. Ces différentes caractéristiques qui renvoient le saut à un acte individuel et singulier, nous semblent cependant limitatives pour considérer la nature de l'activité de l'élève. Elles risquent de restreindre l'implémentation curriculaire à une dimension expérientielle, réductrice selon nous des phénomènes en jeu dans la transmission de savoirs scolaires. En ce sens, la CMP, issue 
de l'analyse de régularités observables en situation de classe veut dépasser la seule étude de cas comme outil d'observation et être un outil de modélisation de l'activité de l'élève au travers des traits saillants que l'activité de l'élève livre à l'observation instrumentée.

\section{Bibliographie}

Amade-Escot, C. (sous la direction de) (2007). Le didactique. Paris: Editions Revue Education Physique et Sport.

Anderson, J.R. (1983). The architecture of cognition. Cambridge, MA : Havard University Press.

Barrué, J-P. (1994). Fonctionnement des savoirs sur l'objet d'enseignement à l'intérieur du système didactique. Thèse de doctorat, non publié. Université Toulouse III.

Borghi, A. M. (2004). Objects concepts and action : Extracting affordances from objects parts. Acta Psychologica, 115, 69-96.

Bouthier, D. (1993). L'approche technologique en STAPS : représentations et actions en didactique des APS. Diplôme d'habilitation à diriger les recherches, non publié. Université Paris-Sud.

Dapena, J. (1980). Mechanics of rotation in the Fosbury-flop. Medecine and science in sports and exercise, 12(1), 45-53.

Davids, K., Handford G., \& Williams, M. (1994). The natural physical alternative to cognitive theories of motor behaviour : an invitation for interdisciplinary research in sports science ? Journal of Sports Sciences, 12, 495-528.

Dietrich, G. (1995). Logiciel 3D vision.

Durand, M., Saury, J., \& Sève, C. (2006). Apprentissage et configuration d'activité : une dynamique ouverte des rapports sujets-environnements. In Barbier, J-M. \& Durand, M., Sujets, activités, environnements, approches transverses (pp 61-84). Paris : Presses Universitaires de France.

Eurydice. (2002). Compétences clés ; un concept en développement dans l'enseignement général obligatoire. Rapport de la Direction Générale de l'Education et de la Culture, Commission Européenne. Consulté en novembre 2007 à http://eurydice.org.

Giordan, A., \& Martinand, J.-L. (1986). Etats des recherches sur les conceptions des apprenants à propos de la biologie. Annales de didactique des sciences, 2. 
Giordan, A., Girault, Y., \& Clément, P. (1994). Conceptions et connaissances. Berne : Peter Lang.

Gouju, J-L. (2001). Objectivation de l'organisation de l'action ; contribution à l'intervention didactique en athlétisme. Thèse de doctorat 3ème cycle, non publié. Université Paris XI.

Gouju, J.L. (2002). L'action comme fenêtre attentionnelle ? Expliciter, 44, 1-9.

Gréhaigne, J.-F., Wallian, N., \& Billard, M. (2005). Approche technologique et modélisation didactique des sports collectifs à l'école. In B. David (coord.) Impulsions 4 (pp. 5980). Paris : INRP.

Kermoal, C. (2008). Les conditions d'une prise en charge scolaire du saut en hauteur ; modélisation de l'activité de l'élève en Fosbury flop. Thèse de doctorat, non publié. Ecole Normale Supérieure de Cachan.

Kermoal, C. \& Gréhaigne, J.-F. (2008). Les espaces subjectifs d'action en Fosbury analyse des discours sur l'action dans la construction motrice personnelle en saut en hauteur chez des élèves de classe de $5^{\text {ème }}$. In N. Wallian, M.-P. Poggi \& M. Musard (eds), Co-construire des savoirs (pp. 271-283). Besançon: Presses Universitaires de Franche-Comté.

Lebeaume, J. (1999). Enjeux et perspectives de la didactique curriculaire : contribution de la didactique de la technologie. Actes des 1ères rencontres scientifiques de I'ARDIST. L'actualité de la recherche en didactique des sciences et des techniques (pp 167-172). Paris : ARDIST.

Martinand, J.-L. (2001). La didactique et les savoirs, Colloque ISPEF/INRP, Les politiques des savoirs, Lyon, 28-29 juin 2001.

Pastré, P. (2004). Le rôle des concepts pragmatiques dans la gestion de situations problèmes : le cas des régleurs en plasturgie. In R. Samurcay \& $\mathrm{P}$. Pastré. Recherches en didactique professionnelle (pp 17-49). Toulouse : Octarès.

Pastré, P. (2005). La conception de situations didactiques à la lumière de la théorie de la conceptualisation dans l'action. In P. Rabardel \& P. Pastré, Modèles du sujet pour la conception, dialectiques, activités, développement (pp 73-107). Toulouse: Octarès.

Pauly, O. (2002). Athlétisme, les erreurs à éviter. Dossier Revue EP.S, 58. Paris : Editions Revue Education Physique et Sport.

Piron, A. (2008). Questions à A. Piron, Apprentissage moteur et intelligence motrice. Revue EP.S, 329, 59-65. 
Poulton, E.C. (1957). On prediction in skilled movements. Psychological Bulletin, 54, 467478.

Récopé, M. (1996). Statut et fonctions du schème de duel dans l'organisation de l'action motrice d'opposition. Thèse de doctorat 3ème cycle. Université Paris V.

Reiffers, J-L. (1997). Accomplir l'Europe par l'éducation et la formation. Rapport du groupe de réflexion sur l'éducation et la formation. Luxembourg : Office des publications officielles des communautés européennes.

Rogalski, J. (2004). La didactique professionnelle : une alternative aux approches de "cognition située" et "cognitiviste" en psychologie des acquisitions, @ctivités, 1 (2), 103-120, http://www.activites.org/v1n2/rogalski.pdf. consulté en mai 2006.

Schmidt, R.A. (1988). Motor Control and Learning : A Behavioural Emphasis, 2ème éd, Champaign, IL : Human Kinetics.

Suchman, L. (1987). Plans and situated action. Cambridge : Cambridge University Press.

Temprado, J-J., \& Montagne, G. (2001). Les coordinations perceptivo-motrices. Paris : Armand Colin.

Theureau, J. (2004). Le cours d'action ; méthode élémentaire. 1 ère édition 1992. Toulouse : Octarès.

Vergnaud, G. (1998). Au fond de l'action, la conceptualisation. In JM. Barbier Savoirs théoriques et savoirs d'action (pp 275-292). Paris: Presses Universitaires de France.

Vermersch, P. (2002). La prise en compte de la dynamique attentionnelle : éléments théoriques. Expliciter, 43, 27-39.

Weill-Fassina, A., Rabardel, P., \& Dubois, D. (1993). Représentations pour l'action. Toulouse : Octarès. 